\title{
Nano Particles of Luminescent Lanthanide Materials
}

Germán E. Gomez ${ }^{1}$, Carlos A. López ${ }^{1}$, R. Lee Ayscue III $^{2}$, María Torres Deluigi, ${ }^{3,4,}$, Karah Knope ${ }^{2}$, Griselda E. $\mathrm{Narda}^{1}$

${ }^{1 .}$ INTEQUI, CONICET, Área de Química General e Inorgánica, FQByF, Universidad Nacional de San Luis, San Luis, Argentina.

2. Georgetown University, Washington, D.C., USA.

3. Departamento de Física, FCFMyN, Universidad Nacional de San Luis, San Luis, Argentina

4. INQUISAL, CONICET, CCT-SL, San Luis, Argentina

* Corresponding author: charo@unsl.edu.ar

The lanthanide ions have fascinating optical properties, including brightness and luminescence. Towards this end, many recent efforts have aimed to develop new or improved optical devices via the pairing of appropriate host materials (chemical compositions) with doped lanthanide ions [1-2]. Trivalent lanthanide ions are characterized by a gradual filling of the $4 f$ orbitals, from $4 f^{0}\left(\mathrm{La}^{3+}\right)$ to $4 f^{14}\left(\mathrm{Lu}^{3+}\right)$. These electronic $[\mathrm{Xe}] f^{\mathrm{N}}$ configurations $(\mathrm{N}=0-14)$ generate a variety of electronic energy levels [3], resulting in intricate optical properties [4]. Consequently, each lanthanide ion exhibits narrow and characteristic $4 f-4 f$ transitions. All $\mathrm{Ln}^{3+}$ ions (except $\mathrm{La}^{3+}$ and $\mathrm{Lu}^{3+}$ ) can produce $f-f$ emissions from ultraviolet to visible and near-infrared ranges. The lanthanide elements manifest this behavior [5], and for this reason they are used in multiple technological applications, such as: lighting devices (economic luminescent lamps, light emitting diodes), television and computer screens, optical fibers, optical amplifiers and lasers.

In this work were successfully synthesized bulk samples of $\mathrm{Ca}_{1-2 \mathrm{x}} \operatorname{Ln}_{\mathrm{x}} \mathrm{Na}_{\mathrm{x}} \mathrm{WO}_{4}$ with $\mathrm{x}=0$ and 0.1 and $\mathrm{Ln}$ $=\mathrm{Eu}, \mathrm{Sm}$ as crystalline white powders, and the structure of the schelite $\left(\mathrm{CaWO}_{4}\right)$ was used as the receiving matrix. For the synthesis a novel mechanical-thermal method of three steps was applied, which included activation, miniaturization by high-energy milling, and further calcination. Then, to obtain the nanoparticles (NP) a fine grinding was carried out. Unlike traditional synthetic routes, this methodology does not use solvent and has a relatively low calcination temperature; so a "green" method to obtain optical materials was achieved.

The nanosized solids were characterized by powder X-ray diffraction, Raman spectroscopy and Scanning Electron Microscopy. The identification and characterization of the solid phases were carried by X-ray Diffraction (XRD). The patterns were refined by the Rietveld method using the FullProf program [6-7]. The profile shape was modelled using the Thompson-Cox-Hastings pseudo-Voigt function [8], and the instrumental resolution parameters were considered in the refinements in order to obtain the microstructural parameters. Furthermore, the apparent size and strain of crystallite is similar in both samples. Obtained strain parameters are high and in accordance with a milling process which generates grain with a distorted surface. The crystallite size of $\sim 18 \mathrm{~nm}$ is greatly reduced with respect to well crystallize samples.

In addition, the Solid-State Photoluminescence (SSPL) was analysed by recording the excitation and emission spectra and calculating the lifetimes from the decay profiles. The materials exhibited red (NPEuCaNaWO$)_{4}$ and orange $\left(\mathrm{NP}-\mathrm{SmCaNaWO}_{4}\right)$ emissions. These results are favorable for the possibility of using these materials in technological applications. Finally, the NP- EuCaNaWO 4 ) was tested as 
chemical sensor towards toxic cations, based on the quenching effect of iron ions through an electrontransfer mechanism [9]. The results are promising for the development of specific chemical sensors by a fast synthesis and "green" methodology.

The morphology of the nanoparticles was analyzed with two diferent Scanning Electron Microscopes (SEM) of Carl Zeiss, the Leo1450VP was first used (some of these micrographs are shown in Figure 1). Subsequently, Field Emission Scanning Electron Microscope (FE-SEM) in lens $\Sigma$ igma was accessed, Figure 2 shows some images obtained with this microscope. It was observed that the NPs have a spherical shape, and that the diameters are on average $300 \pm 50 \mathrm{~nm}$ and $150 \pm 20 \mathrm{~nm}$ when the doping is $\mathrm{Sm}$ and $\mathrm{Eu}$, respectively [10].

\section{Referencias}

[1] C D S Brites et al., New J. Chem. 35 (2011), p. 1177.

[2] L D Carlos, Adv. Mater. 21 (2009), p. 509.

[3] E G Moore, A P S Samuel and K N Raymond, Acc. Chem. Res. 42 (2009), p. 542.

[4] J C G Bünzli, Chem. Rev. 110 (2010), p. 2729.

[5] J Liu et al., Dalton Trans. 45 (2016), p. 12094.

[6] H M Rietveld, J. Appl. Crystallogr 2 (1969), p. 65.

[7] J Rodríguez-Carvajal, Phys. B Condens. Matter 192 (1993), p. 55.

[8] P Thompson, D E Cox and J B Hastings, J. Appl. Crystallogr. 20 (1987), p. 79.

[9] Germán Gomez et al., Dalton Trans. 48 (2019), p. 12080

[10] The authors thank the services provided by the following Argentine Microscopy Laboratories: LABMEM (Universidad Nacional de San Luis) and LAMARX (Universidad Nacional de Córdoba).
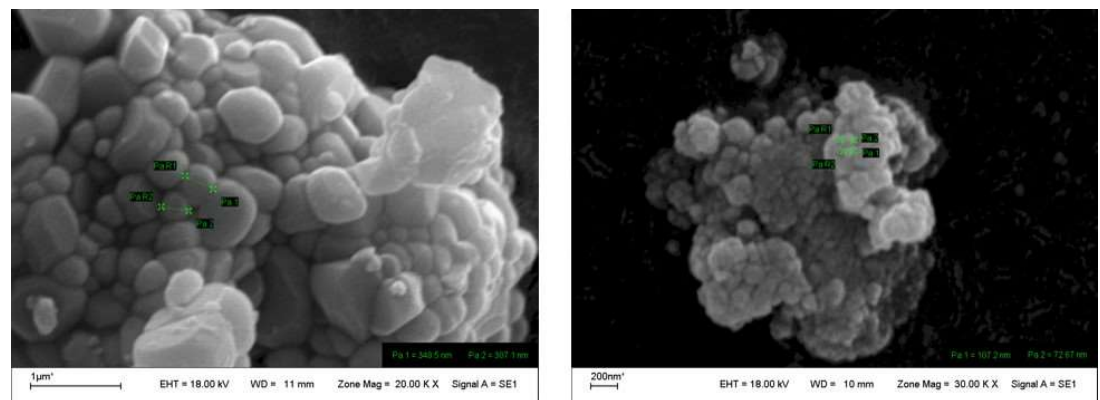

Figure 1: Nano particles of Sm (left) and Eu (right) obtained whit SEM Leo 1450VP
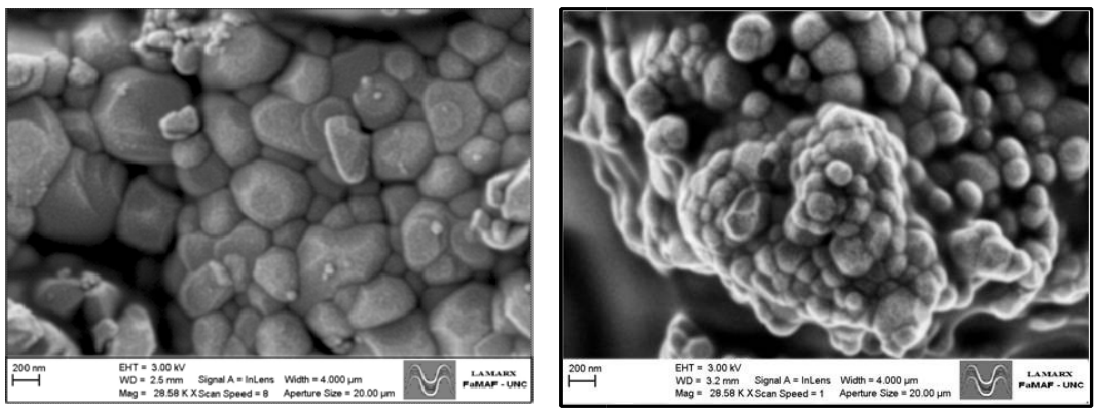

Figure 2: Nano particles of Sm (left) and Eu (right) obtained whit FE-SEM in lens Eigma. 